\title{
Religionsverzicht um des guten Lebens willen?
}

\author{
Eine skeptische Übung
}

Andreas Urs Sommer

One considerable advantage that arises from Philosophy, consists in the sovereign antidote which it affords to superstition and false religion. ${ }^{1}$

Dass man trotz eines Verzichts auf Religion ein mit den gängigen moralischen Vorstellungen übereinstimmendes Leben führen könne, ist eine Vermutung, mit deren Äusserung sich Pierre Bayle vor rund 330 Jahren beträchtliche Schwierigkeiten eingehandelt hat. Dass Atheismus kein verabscheuungswürdiges moralisches Übel zu sein braucht, ${ }^{2}$ ist eine Einsicht, die sich trotz der Erschlaffung religiöser Inbrunst in weiten Teilen Europas bisher noch nicht überall durchgesetzt hat. Entgegen einer in unseren Breiten anhaltenden Tendenz, der christlichen Religion in ihrer kirchlich institutionalisierten Form den Rücken zu kehren, werden doch gegenwärtig wieder Anzeichen einer Renaissance des Religiösen beschworen. Solche Anzeichen könnten die Behauptung nahelegen, der Atheismus stelle keine lebbare Daseinsoption dar. Wie immer Religionssoziologinnen und Theologen die empirischen Befunde zu Säkularisierung und Resakralisierung interpretieren, so ist doch deutlich, dass aus solchen empirischen Befunden normativ nichts folgt: Weder spräche es für den Atheismus, wenn sich die Mehrheit der Bevölkerung zu ihm bekennen sollte, noch spräche es für die Kirchen, wenn es ihnen erneut gelänge, diese Mehrheit hinter sich zu scharen. Eigentümlich ist aber, dass militanter Atheismus, wie er sich noch in den sechziger und siebziger Jahren gegen den theologisch-politischen Komplex machtvoll artikulierte, heute im deutschsprachigen Raum wenigstens in die Nischen weltanschaulicher Sondergruppen abgewandert ist. Trotz des vermehrten Auftretens militanter Religion in

1 D. Hume: Essays on Suicide and the Immortality of the Soul. Never before published. With Remarkes, intented as an Antidote to the Poison contained in these Performances, by the Editor, London 1783,1.Was folgt aus dieser Zweckbestimmung der Philosophie, wenn man probehalber einmal annimmt, dass es überhaupt keine richtige Religion gebe?

2 Vgl. [P. Bayle], Pensées diverses, ecrites à un Docteur de Sorbonne, à l'occasion de la Comete qui parut au mois de Decembre 1680. Quatrieme Edition, Bd. 1, Rotterdam 1704, \ 105, S. 214. 
Gestalt von Fundamentalismen scheint Religionskritik hierzulande (sehr im Unterschied zu anglo- und frankophonen Ländern ${ }^{3}$ ) kein vordringliches weltanschauungskämpferisches Traktandum mehr zu sein. Hat mit der schwindenden Dominanz kirchlicher Institutionen in Westeuropa auch der Religionskritik - die im 20. Jahrhundert über weite Strecken eine Kritik an kirchlichmoralischen Macht- und Unterdrückungsmechanismen war - das Sterbeglöckchen geläutet? Religion, die nicht mehr in Gestalt einer freiheitsberaubenden Zwangsanstalt erscheint, bietet für eine Kritik, die das Existenzrecht der Religion prinzipiell bestreitet, nur noch wenig Angriffsfläche. Eine Religionskritik, die die Vernichtung der Religion und ihre Ersetzung beispielsweise durch Wissenschaft will, droht sich in dem Augenblick zu erledigen, wo Religion ihrer eigenen Omnipotenzphantasien entsagt. Religionskritik müsste dann womöglich etwas anderes sein, wenn sie überhaupt noch sein will - nicht länger jedenfalls die Handlangerin unbändiger, aber selbst sehr dogmatischerVernichtungswünsche.

Vielleicht könnte ja das Verhältnis von Religionskritik und Religion in Analogie zur Kunstkritik und Kunst neu bestimmt werden: Kunstkritik trägt - zumindest gewöhnlich - auch nicht die Vernichtung der Kunst im Sinn.

Wenn ich hier darüber nachdenke, ob der Verzicht auf Religion ein gutes Leben befördern kann, damit also in die Rolle des advocatus diaboli schlüpfe und Sie als Leser zu einer skeptischen Übung nötige, ${ }^{4}$ dann ist dies schon Zumutung genug. Ich werde es mir daher versagen, Sie mit der Rekapitulation atheistischer und religionskritischer Positionen der Vergangenheit zu langweilen. Stattdessen will ich mich der scheinbar einfachen Frage zuwenden, inwiefern Religionsverzicht um des guten Lebens willen gegenwärtig eine vertretbare oder vielleicht eine gebotene Haltung ist, ohne dabei wie beispielsweise Michel Onfray, Richard Dawkins oder Daniel Dennett einen atheistischen Kreuzzug zu führen. ${ }^{5}$ In einem ersten Abschnitt

\footnotetext{
$3 \mathrm{Zu}$ den soziologischen Gründen, die den militanten angloamerikanischen Atheismus (vgl. unten Anm. 5) zu erklären helfen, siehe F. Unger, Säkularer (In-)Humanismus. Agnostizismus und Atheismus in der "christlichen Nation" Amerika, in: R. Faber/S. Lanwerd (Hrsg.), Atheismus. Ideologie, Philosophie oder Mentalität?, Würzburg 2006, S. 249-269.

4 Vgl. A.U. Sommer, Die Kunst des Zweifelns. Anleitung zum skeptischen Denken, München $2005,{ }^{2} 2007$.

5 Der militante angloamerikanische Atheismus, der während der letzten Jahre im Namen der "Brights», der "naturalistisch" Erhellten für viel Aufregung sorgte, hat für nuancenbewusste Kontinentaleuropäer etwas Peinliches. Verglichen mit Schriften wie R. Dawkins' The God Delusion, London 2006 oder S. Harris' The End of Faith.
} 
möchte ich zu klären versuchen, worüber wir reden, wenn wir von Religion und gutem Leben reden. Ein zweiter Abschnitt wird erörtern, was Religionsverzicht für den multizentrischen Menschen der Moderne angeraten sein lassen könnte. Ein dritter Abschnitt wird schliesslich einige unvorgreifliche Schlussfolgerungsmutmassungen anstellen.

\section{I.}

Philosophen lieben Allgemeinbegriffe mitunter mehr als die Weisheit, zu deren Liebe sie sich in ihrer Funktionsbezeichnung bekennen. Deshalb erwartet der Leser eines philosophischen Beitrages womöglich einen abstrakten Begriff von Religion. Aber ich schrekke davor zurück. Will man die Fülle des landläufig unter Religion Firmierenden zu einem abstrakten Begriff verdünnen, würde als vorläufige Bestimmung, was man unter Religion verstehen kann, so etwas herauskommen wie: eine Lebensansicht, eine das Leben bestimmende Haltung, die dieses Leben und diese Welt ins Verhältnis $\mathrm{zu}$ einem Überweltlichen, Unbedingten, Grundlegenden bringt. Dabei ist bekanntlich die Vorstellung eines persönlichen Gottes oder anthropomorpher Götter keine notwendige Voraussetzung für das unter »Religion" Gefasste.

Wenn ich hier die Chancen und Risiken eines Verzichtes auf Religion erwäge, dann orientiere ich mich jedoch nicht an einem derartig abstrakten Annäherungsbegriff von Religion in grösstmöglicher Unterbestimmtheit, sondern an der für Europa kulturell prägenden Religion, am Christentum also. Ungeachtet des seitens christlicher Theologen gelegentlich vorgebrachten, mir nie ganz verständlichen Einwandes, das Christentum sei gar keine Religion, soll hier zurVereinfachung der Aufgabe das Christentum den Idealtypus dessen darstellen, was Religion ausmacht. Damit sei jedoch nicht - wie etwa bei Ludwig Feuerbach im Gefolge Hegels - behauptet,

Religion, Terror, and the Future of Reason, New York 2004, lesen sich beispielsweise P. H. Thiry d'Holbachs Théologie portative ou Dictionnaire abrégé de la Religion chrétienne, 1768, oder F. Nietzsches Der Antichrist wie Meisterwerke der Differenzierungskraft und Subtilität. Ein atheistisches Bekenntnis kommt offenbar nicht ohne strengen Dogmatismus aus, was einen daran zweifeln lässt, wie konsequent Atheismus gedacht werden kann, ohne selbst zur doktrinären Religion zu werden (vgl. z. B. auch K. Joël, Der Glaube der Atheisten, in: Ders., Antibarbarus. Vorträge und Aufsätze, Jena 1914, 174-191). Einen anschaulichen tour d'horizon in der Landschaft gegenwärtiger Atheisten gibt G. Wolf, Hört endlich auf zu glauben, in: Das Magazin (Tagesanzeiger Zürich), Nr. 2, 13. Januar 2007, 16-25. 
das Christentum sei die reinste, vollkommenste Ausprägung des Begriffes von Religion (die idealistische Vorstellung, ein Begriff würde sich empirisch ausprägen, ist mir fremd). Vielmehr ist diese Orientierung am Idealtypus Christentum erstens dem Umstand geschuldet, dass das Christentum in meinem eigenen Fall die einzige Religion ist, von der ich nicht bloss angelesene und durch distanzierte Beobachtung gewonnene, sondern konkrete, lebenspraktisch fundierte Anschauung habe. Und zweitens, wichtiger noch, dem Umstand, dass die Vorstellung dessen, was Religion ausmacht und ausmachen soll, in unserem Kulturkreis trotz aller Entsakralisierungen nach wie vor vom Christentum bestimmt wird. Wenn ich von Religionsverzicht spreche, so vermag ich nur im Hinblick auf eine konkrete Religion, wie sie das Christentum darstellt, zu ermessen, was der Verzicht darauf bedeuten könnte.

Dennoch aber sollte man sich bei diesem Verfahren, vom Christentum her eineVorstellung davon zu gewinnen, was Religion ausmacht, um zu prüfen, worauf man verzichtet, wenn man auf Religion verzichtet, nicht allzu sehr von den historisch-dogmatischen Spezifika des Christentums leiten lassen. So wird hier nicht nur ausgeblendet, dass das Christentum der Urgemeinde sich sehr von dem unterschied, was Origenes, Augustinus, Johannes Damascenus, Thomas von Aquin, Martin Luther, Pius IX. oder Karl Barth unter "Christentum« verstanden haben. Es werden auch die dogmatischen Besonderheiten Monotheismus, Schrift- und/oder Liebesreligion, ${ }^{6}$ Trinitätslehre, Christologie, Leidenstheologie, Eschatologie, Ekklesiologie - tunlichst aussen vor bleiben. ${ }^{7}$ Ohne gewisse Abstraktionen komme ich, um der Frage willen, also doch nicht aus. Was aber als Spezifikum von Religion nach Maßgabe des Christentums hier festgehalten wird, ist der umfassende Anspruch von Religion: Religion nach christlichem Muster will letzte Instanz der Orientierung, erste und letzte Bezugsgrösse des menschlichen Lebens sein; sie will alle anderen möglichen Gesichtspunkte der Lebensorganisation ihrem Gesichtspunkt unterordnen. Dieser Gesichtspunkt ist der einer überweltlichen Lenkung des Weltgeschehens. Damit relativiert Religion alle rein irdischen Belange - so hört beispielsweise Politik

\footnotetext{
6 A.Angenendt, Toleranz und Gewalt. Das Christentum zwischen Bibel und Schwert, Münster 2007, erinnert an die zivilisatorische Kraft der Liebesreligion.

7 Die von B. Russell, Warum ich kein Christ bin. Ein Vortrag, in: Ders., Warum ich kein Christ bin. Über Religion, Moral und Humanität, Hamburg 1968, 17-34, hier 18 , angeführten drei Kriterien des Christseins, nämlich der Glaube an Gott, an die Unsterblichkeit und an Christus, dienen nur dazu, die altbekannten religionskritischen Topoi abzuraspeln - ein Verfahren, das ich hier nicht wiederholen will.
} 
auf, etwas Letztes und Absolutes sein zu können und sein zu müssen. ${ }^{8}$ Religion identifiziert das für sie objektiv Letzte und Absolute mit einem Überweltlichen, im Christentum »Gott» genannt. Religion in diesem Sinne macht alles Irdische von einem Überirdischen abhängig. Diese Abhängigkeit wird artikuliert in der Diagnose, dass diese Welt und die in ihr lebenden Menschen erlösungsbedürftig seien. Erlösung ist in altabendländischer Terminologie das Angebot, das Religion für die Menschen bereithält.

Verzichtet man bei der Betrachtung des Begriffes des guten Lebens darauf, die Jahrtausende geistiger Arbeit zu resümieren, die sich um ihn ranken, so fällt zunächst auf, dass er keineswegs notwendig mit dem Begriff des Glücks identisch zu sein braucht. ${ }^{9}$ Wenn Glück, abgesehen vom bloss zufallenden Glück, einen Zustand der Vollkommenheit, der Übereinstimmung von Sein und Sollen meint, kann demgegenüber "gutes Leben" auch bloss bedeuten, dass sich dieses Leben an einem wie auch immer beschaffenen Sollen ausrichtet, womit über das Sein noch nichts gesagt ist. Eingefleischte Stoiker zum Beispiel werden zwar behaupten, dass der moralisch Gute, der Tugendhafte notwendig glücklich sei. Aber ein solcher Glücksbegriff ist dem modernen Publikum nur noch schwer zu vermitteln. Klammern wir hier zur Komplikationsvermeidung den Glücksbegriff also aus und bestimmen "gutes Leben« zunächst nur als ein Leben, das den in es gesetzten Ansprüchen und Erwartungen entspricht. Dabei ist entscheidend, wer oder was diese Ansprüche und Erwartungen definiert.

Gewiss kann man davon sprechen, dass historisch konkrete Religionen historisch ebenso konkreteVorstellungen davon ausgebildet haben, was die Güte eines konkreten Menschenlebens ausmacht etwa ein Leben, das sich peinlich genau nach den Geboten dieser konkreten Religion richtet (soweit dies überhaupt für menschenmöglich erachtet wird), oder, wie im Christentum, ein Leben, das durch Christi Heilstat gerechtfertigt, damit gut gemacht worden ist. Es ist klar, dass ein allfälliger Religionsverzicht mit einer derartigen religiösen Bestimmung guten Lebens nicht zu vereinbaren wäre.

Interessanter erscheint der Fall, der die Definitionsmacht darüber, was ein gutes Leben ist, nicht einer überindividuellen Instanz wie der

\footnotetext{
8 Vgl. H. Ottmann, Athen und Jerusalem - eine Umwertung antiker Werte durch das christlich-jüdische Denken?, in: M. Brocker/T. Stein (Hg.), Christentum und Demokratie, Darmstadt 2005, 29-37, hier 30 .

9 Vgl. z. B. G.H. von Wright, The Varieties of Goodness, London/New York 1963, 88 Aristoteles, Nikomachische Ethik, $1095 \mathrm{a} 19$ und 1098b21, scheint demgegenüber das eu zen mit der eudaimonia zu identifizieren.
} 
Religion oder der `herrschenden gesellschaftlichen Morak überlässt, sondern sie vielmehr dem Individuum zuweist, das das Leben lebt, über dessen Güte zu befinden ist. Wenn es das Individuum ist, das darüber entscheidet, inwiefern sein eigenes Leben ein gutes Leben ist, dann ist es durchaus möglich, dass dieses Individuum um der Güte seines Lebens willens auf Religion verzichten zu sollen glaubt. Jedoch ist diese Erkenntnis einigermaßen trivial. Jemand, der um seines guten Lebens willen auf Religion verzichten will, müsste sich die Frage gefallen lassen, ob es für diesen Verzicht Argumente beibringen kann, die über individuelle Präferenzen und Idiosynkrasien hinausreichen. Gutes Leben muss offenbar auch überindividuell verantwortbar sein.

\section{II.}

Ist Religion nach altabendländischem Verständnis die Sachwalterin der Erlösung, dann liegt hier auch ein Angelpunkt der Frage nach Religionsverzicht. Denn gleichgültig, ob man >Religionsverzicht $<$ als eine deskriptive oder als eine normative Kategorie verwendet - ob man also die westeuropäische Gegenwart faktisch von Religionsverzicht bestimmt sieht oder aber einen solchen Religionsverzicht für angeraten hält -, ist schwerlich zu verkennen, dass das Wissen darum, was und wozu Erlösung sein soll, der Furie des Verschwindens anheimgefallen ist. Der Mensch hat in der Moderne - nach einigen glücklosen Versuchen, sich mittels politischer Massenideologien Ersatzerlösungen zu verschaffen - nach und nach verlernt, sich erlösungsbedürftig zu fühlen. Daran ändern auch die Unkenrufe nichts, dieser Mangel an gefühlter Erlösungsbedürftigkeit sei gerade ein Beweis für die Weltverfallenheit und damit eben erst recht für die faktische Erlösungsbedürftigkeit der Menschen. Dem Gegebenen Tribut zollend, sind viele, namentlich protestantische Kirchenvertreter längst dazu übergegangen, Erlösung aus ihrem Vokabular zu streichen und ganz auf die Diesseitigkeit ihres Christentums zu pochen. Manch einer wird sich vielleicht mit Wehmut daran erinnern, dass es dort, wo jetzt »Singen mit Bruder Jesus« oder »Deckchenhäkeln für Lambarene« angesagt ist, einst um das ewige Heil und die ewige Verdammnis ging - und dass dieses altbackene Christentum nichts Nettes, nichts verzärtelt `Humanistisches` hatte.

Das schwindende Gefühl der Erlösungsbedürftigkeit hat unmittelbar damit zu tun, dass die Frage nach einem Leben nach dem Tod wie kaum eine andere Frage sich gegenwärtig als eine kulturell 
kontingente Frage erweist ${ }^{10}$ - und zwar nicht nur in den darauf gegebenen Antworten, sondern als Frage selbst. Man gerät in diese Frage nicht mehr so leicht hinein; sie widerfährt einem in der Gegenwart nicht mehr einfach - wobei die Frage nach einem Leben nach dem Tod keineswegs zu verwechseln ist mit der Frage nach der Sterblichkeit, der man auch in der Gegenwart nicht entkommt. ${ }^{11}$ Nichts und niemand hindert den Menschen in der Moderne, die Frage nach einem Leben nach dem Tod für radikal gleichgültig und sich selbst für ein Lebewesen zu halten, das wie alle anderen Lebewesen zu einem unwiderruflichen, biologisch vorgegebenen Ende bestimmt ist.

Eine solche Einsicht kann ganz frei von Schrecken sein, verlagert zugleich aber das Schwergewicht von einem erhofften Jenseits in das Diesseits. Wenn dieses irdische Leben das einzige ist, das wir haben, dann tun wir gut daran, es zu nutzen. Wenn heutzutage medizinisch so viel für dieVerlängerung des einen irdischen Lebens getan werden kann, ist dies gerade kein Ausdruck eines parareligiösen Wunsches nach Unsterblichkeit, sondern zeigt nur an, dass uns an diesem irdischen Leben offensichtlich so viel liegt, dass wir es möglichst ausdehnen wollen.

Es könnte also sein, dass sich in der Moderne bei vielen Menschen das Bedürfnis nach Erlösung ebenso sehr verflüchtigt wie der Wunsch, ein Absolutes als geglaubtes Gegenüber zu haben. Die Resten solcher Bedürfnisse hat man ins Reich der Fiktion, in Film und Belletristik ausgelagert, wohl wissend, es dort nicht mit Realia, sondern Irrealia (wahlweise Surrealia), nicht mit Facta, sondern Ficta zu tun zu haben. Freiheit könnte in der europäischen Gegenwart gerade darin bestehen, sich von den Zwängen der Selbstaufhebung des Menschen - nämlich als nichtswürdiger Sünder - gleichermaßen freigemacht zu haben wie von den Zwängen der Selbstverabsolutierung des Menschen. Derartige Selbstverabsolutierung wird in der christlichen Vorstellung eines menschgewordenen Gottes ebenso anschaulich wie in der postchristlichen Vorstellung eines Über- oder Edelmenschen.

Religionsverzicht könnte also Verzicht auf hypertrophe Sinnansprüche bedeuten. Es braucht kein Ausdruck von Kleinmütigkeit zu sein, sondern könnte für Klugkeit sprechen, wenn man die Idee eines letzten grossen Sinns, einer letzten, absoluten Bezugsgrösse

${ }^{10}$ Vgl. z.B.A.U. Sommer, Das eigene Sterben denken, in: Ch. Burbach/F. Heckmann (Hg.), Übergänge. Annäherungen an das eigene Sterben, Göttingen 2011, 148-155.

${ }_{11}$ Dazu ausführlich A.U. Sommer, Die Kunst der Seelenruhe. Anleitung zum stoischen Denken, München 2009. 
fallenlässt. Es gibt auch keinen Grund, weshalb man ob des Verlustes einer solchen Bezugsgrösse noch wie Nietzsche und manche Existenzialisten in Ekstasen der Verzweiflung und des Ekels geraten oder auf die Schaffung einer diesseitigen, absoluten Bezugsgrösse sinnen sollte. Die Geburtswehen des modernen Menschen als eines multizentrischen Wesens waren gewiss heftig - aber jetzt bedarf er für sein Dasein vielleicht keines letzten Zentrums mehr. ${ }^{12}$

Der multizentrische Mensch muss nicht mehr über sein Dasein oder dessen Endlichkeit getröstet werden. Die erste Frage des Heidelberger Katechismus, um die herum sich die ganze christliche Religion organisiert, nämlich »Was ist dein einziger Trost im Leben und im Sterben?«, ist eine Frage, die der moderne multizentrische Mensch nicht mehr versteht. Die Rede von einer Renaissance des Religiösen täuscht leicht darüber hinweg, dass sich das, wofür Menschen heute Trost suchen, weitgehend individualisiert hat. Weil die Drohung der Verdammnis nicht mehr sticht, müssen wir nur noch darüber getröstet werden, den Liebsten, den Job, den Kanarienvogel oder die Altersrente verloren zu haben. Natürlich kann Religion hier tröstend einspringen, läuft aber Gefahr, bald von wirkungsvolleren, nämlich pragmatischen Mitteln zur Problembewältigung ausgehebelt zu werden.

Mit anderen Worten: Es könnte sein, dass wir die Probleme nicht mehr haben, auf die die Religion die Antwort war - und ob sie Antwort auch auf andere Fragen sein kann, ist offen. Mit der sinkenden Nachfrage nach Erlösung droht Religion funktionslos $\mathrm{zu}$ werden. Das hat zur Folge, dass sich Religion neue Betätigungsfelder, neue Funktionen sucht - wie man es beispielsweise am mittlerweile auch schon wieder verblichenen, friedensbewegten "Demonstrationsprotestantismus " beobachten konnte. ${ }^{13}$ Man braucht diese Suche der Religion nach neuen Funktionen nicht als Abirrung vom wahren Sinn der Religion zu geisseln; sie gründet in einem legitimen Selbstbehauptungsinteresse. Und es ist ja keineswegs so, dass Religion sich erst heute neue Funktionen sucht: Der Frankenfürst Chlodwig beispielsweise hat, wollen wir Gregor von Tours trauen, das Christentum mitnichten angenommen, weil er

\footnotetext{
12 Zum Thema vgl. z. B. R. Rorty, Der Vorrang der Demokratie vor der Philosophie, in: Ders., Solidarität oder Objektivität? Drei philosophische Essays, Stuttgart 1988, 82-125, hier 99.

${ }^{13}$ J.H. Claussen, Zurück zur Religion. Warum wir vom Christentum nicht loskommen, München 2006, 187.
} 
danach lechzte, derVerdammnis zu entgehen, sondern weil er einen Krieg gewinnen wollte. ${ }^{14}$

Aber der multizentrische Mensch der Moderne scheint keine Verwendung mehr zu haben für einen Gott, der ihm Erlöser sein will - ebenso wenig für einen Gott als Beistand in den alltäglichen Kriegen des Daseins. Er weiss nicht mehr wirklich, was er mit Religion anstellen soll, denn ein Unbedingtes erscheint ihm angesichts all dessen, was sich als bedingt erweist, als eine höchst unsichere und als eine nicht wirklich wünschenswerte Sache. Das "Gefühl schlechthinniger Abhängigkeit«, auf dem Schleiermacher bekanntlich die Religion begründet wissen wollte, ${ }^{15}$ ist ein Gefühl, das dem multizentrischen Menschen fremd geworden ist - ohne dass er sich deshalb wie Hegel zu polemischen Ausfällen hinreissen liesse. ${ }^{16}$ Dem multizentrischen Menschen ist Religion kaum mehr als etwas, was anlässlich von Weihnachtsgottesdiensten oder Oratorienaufführungen für sporadische Gefühlsaufwallungen, für einen gelegentlichen Erhabenheitskitzel sorgen kann - etwas, was den verführerischen Reiz derVerunklarung verströmt, den man sich zu hohen Feiertagen gönnt. Kurz: Religion ist dem multizentrischen Menschen nur noch ein Ornament.

III.

Wenn ich es zunächst offen gelassen habe, ob ich bei "Religionsverzicht« über ein Sein oder ein Sollen schreibe, ist mittlerweile wohl deutlich geworden, dass Religionsverzicht vielfach ein Faktum ist. Religionsverzicht bedeutet zunächst, dass man auf die religiösen, namentlich christlichen Antworten zu verzichten gelernt hat. ${ }^{17}$ Und

\footnotetext{
${ }^{14}$ Gregor von Tours, Historiarum libri decem II 30 (hg. v. R. Buchner, Bd. 1, Darmstadt 1967, 116)

15 Vgl. F. Schleiermacher, Der christliche Glaube nach den Grundsäzen der evangelischen Kirche im Zusammenhange dargestellt, 5. unveränderte Ausgabe, Bd. 1, Berlin 1861, Einleitung $\ 4,15-22, .$.

16 "Gründet sich die Religion im Menschen nur auf ein Gefühl, so hat solches richtig keine weitere Bestimmung, als das Gefühl seiner Abhängigkeit zu sein, und so wäre der Hund der beste Christ, denn er trägt dieses am stärksten in sich und lebt vornehmlich in diesem Gefühle. Auch Erlösungsgefühle hat der Hund, wenn seinem Hunger durch einen Knochen Befriedigung wird." G.W. F. Hegel,Vorrede zu Hinrich's Religionsphilosophie [1822], in: Berliner Schriften 1818-1831 = Werke, Bd. 11, Frankfurt a.M. 1986, 42-67, hier 58 .

${ }_{17}$ Damit würde auch ein »Ersatz« für das Christentum überflüssig (vgl. G. Wyneken, Abschied vom Christentum. Ein Nichtchrist befragt die Religionswissenschaft, Ham-
} 
allmählich zeichnet sich auch ab, dass man auf die religiösen Fragen ohne jede heroische Anstrengung ebenfalls verzichten kann, falls man das will - jeder nach seiner Façon. ${ }^{18}$

Man könnte nun vermuten, mit dem Zahmwerden des Christentums sei die Religionskritik ebenfalls zahm geworden. Religion verschwindet in Europa nicht, aber sie hat sich mehr und mehr vergleichgültigt, weswegen sie keiner scharfen Kritik mehr zu bedürfen scheint. Wenn ich hier frage, ob ein Verzicht auf Religion nicht nur empirisch $\mathrm{zu}$ beobachten sei, sondern auch um eines guten Lebens willen geboten sein könnte, dann meine ich damit den Verzicht auf Religion im altabendländischen Sinne. Es scheint mir keine ganz abwegige Forderung zu sein, um eines guten Lebens willen auf Religion als etwas zu verzichten, was das ganze Leben in Beschlag nimmt und auf ein Überirdisches hin ausrichtet. Faktisch haben auf Religion in dieser Bedeutung bereits all jene Kirchenmitglieder Verzicht geleistet, die zwar brav ihre Kirchensteuern bezahlen und den Weihnachtsgottesdienst besuchen, sonst aber alles Religiöse nicht länger selbst leben, sondern an ihre Kirche delegieren. ${ }^{19}$

Die Lauheit der Seelen, die viele Kirchenvertreter brandmarken, erscheint unter leicht justierter Perspektive als eine schwer erkämpfte, intellektuelle Errungenschaft der Moderne. ${ }^{20}$ Diese Lauheit bedeutet Freiheit von aufs Ganze gehenden Entscheidungszumutungen - auch von den Zumutungen einer als lebensbestimmend missverstandenen Religion.

Vielleicht liegt ja die Chance der Religion gerade in ihrer Vergleichgültigung - vielleicht sollte sie aufhören, à tout prix zu den Essentialia des Lebens gehören zu wollen.Vielleicht reicht es, wenn

burg 1970, 254).

${ }^{18}$ Womöglich hätte heute eine "gottlose Mystik«, »ledig des Ichwahns«, wieder eine Chance, wie sie F. Mauthner, Der Atheismus und seine Geschichte im Abendlande, Bd. 4, Stuttgart/Berlin 1923, 446 skizziert. »Nach rückwärts blickend ist Sprachkritik alles zermalmende Skepsis, nach vorwärts blickend, mit Illusionen spielend, ist sie eine Sehnsucht nach Einheit, ist sie Mystik. (447).

${ }_{19}$ Vgl. auch Claussen, Zurück zur Religion, S. 79 u. passim.

${ }^{20}$ Entsprechend altbacken wirken heute schroff religionskritische Schriften. T. Mosers Gottesvergiftung, Frankfurt a.M. 1976, beispielsweise liest sich wie ein Buch, das aus einer ähnlich fremden Welt stammt wie die Imitatio Christi des Thomas a Kempis. Moser beutet in seiner Gottesanklage eine fromme Inbrunst aus, die sich nur durch eine zufällige Richtungsänderung des Affekts nicht in Selbstgeisselung und äusserster asketischer Praxis ausmünzt. Mosers Pamphlet ist als ein Gebet, eine Verfluchung konzipiert - analog zu Augustins Confessiones. Wie kann Gott als so real, Religion als so essentiell empfunden werden, fragt sich der durchschnittlich säkularisierte Leser heute. 
sie Ornament eines multizentrischen Lebens ist. Warum es mit der Religion nicht so halten wie die Römer in der späten Republik oder Lukian in den Göttergesprächen?

Man könnte,Vorschlag zur Güte, gerade in derVerzichtbarkeit der Religion, ein Argument dafür sehen, sich für sie, in altabendländischem Sinne zu entscheiden. Nur wenn ich die Wahl habe, Religion auch lassen zu können, ist sie wertvoll. Aber der multizentrische moderne Mensch wird es vielleicht um seines guten Lebens willen beim Entscheidungsverzicht in religiösen Dingen belassen. ${ }^{21}$ Es gibt für ihn keine Nötigung mehr, sich auf eine vorgeblich letzte Orientierungsinstanz einzulassen. Ja, er wüsste, sollte er sich für Religion entscheiden, vielleicht nicht einmal, wofür er sich da eigentlich entscheidet. Religion im altabendländischen Sinne will Entscheidung. ${ }^{22}$ Aber, so scheint es, Entscheidung für etwas, für das man sich nicht mehr entscheiden kann - weil man nicht mehr weiss, was es ist, für das man sich entscheidet. Die Entscheidung, die die sichtbare Religion immer noch verlangt, wird daher vom multizentrischen Menschen suspendiert werden. Er oder sie wird auf eine Religion verzichten, die Bekenntnisse verlangt, und wird stattdessen lebenspraktisch diverse religiöse Optionen ohne Bekenntnisdruck und je nach jeweiligen Präferenzen durchspielen. Gelassene, selbstreflexive Religiosität als flüchtige Vorabendstimmung könnte da entstehen. ${ }^{23}$ Es gibt keine Nötigung zu Letztentscheidungen mehr; wir brauchen keine Wahl mehr treffen, die uns zur Religion nötigt, denn es geht nicht mehr länger um Fragen des ewigen Heils und derVerdammnis.

Die Frage nach dem Verzicht auf Religion um des guten Lebens willen führt mich also zu einem Plädoyer für eine

\footnotetext{
${ }^{21}$ Immerhin könnte man die Vorstellung, dass selbstbestimmtes Glück der oberste Zweck eines guten Lebens sein soll, in unauflösbarem Widerspruch zu fremdbestimmten Heil als oberstem Zweck eines religiösen Lebens stehen sehen. E. Angehrn, Der Begriff des Glücks und die Frage nach der Ethik, in: Philosophisches Jahrbuch, 92 (1985), 35-52, hier 50, stellt die "Selbstzweckstruktur des Wollens" als Zentrum des Glücksbegriffs heraus. Die religiöse Heilssehnsucht, so würde ich im Rahmen unserer Fragestellung ergänzen, will gerade nicht das Wollen, sondern dessen Abschaffung und Überwindung. Sie will ein Anderssein.

${ }^{22} \mathrm{Vgl}$. Claussen, Zurück zur Religion, 48.

${ }^{23}$ Vor ein paar Jahren machte sich J. Habermas, Ein Bewusstsein von dem, was fehlt. Über Glauben und Wissen und den Defaitismus der modernen Vernunft, in: Neue Zürcher Zeitung, 10. Februar 2007, für »ein Reflexivwerden des religiösen Bewusstseins angesichts der Notwendigkeit, die eigenen Glaubenswahrheiten sowohl zu konkurrierenden Glaubensmächten wie zum Monopol der Wissenschaften auf die Produktion von Weltwissen in Beziehung zu setzen", stark und mahnte an, die moderne Vernunft solle sich auf ihre altabendländisch-religiösen Wurzeln besinnen.
} 
Entkonfessionalisierung der Religion im wortwörtlichen Sinn. D. h. Religion soll nicht mehr bekenntnisgebunden sein, sondern ein - in erster Linie dekoratives Element - eines sich redlich mit dem Leiden herumschlagenden, multizentrischen Lebens, das vielleicht nicht "gut" ist, aber das Beste herausholen will. ${ }^{24}$ Religion gibt in diesem multizentrischen Leben keine Letztorientierungen mehr, sondern ist eine von zahlreichen im selben Leben möglichen, gleichberechtigten Weisen, das Vorläufige zu organisieren.

Unter postaufklärerischen Bedingungen überfordert man Religion, will man von ihr letzte Gewissheiten. Erlösung von Erlösung, auch von der Idee der Erlösung, könnte sich leicht einstellen. Die Kosten eines so verstandenen Religionsverzichts hielten sich dabei vermutlich in Grenzen. Sie bestünden nur in einer gewissen Ehrlichkeit, die Dinge so zu sehen, wie sie sind. Als Verzicht auf letzte Festschreibungen findet sich Religionsverzicht im kürzesten Jesus-Logion aus dem apokryphen Thomas-Evangelium verdichtet: "Werdet Vorübergehende! ${ }^{25}$

- Prof. Dr. Andreas Urs Sommer ist wissenschaftlicher Kommentator an der Forschungsstelle "Nietzsche-Kommentar der Heidelberger Akademie der Wissenschaften an der Albert-Ludwigs-Universität Freiburg.

${ }^{24}$ Vgl. auch S. Richter, Lob des Optimismus. Geschichte einer Lebenskunst, München 2009 .

${ }^{25}$ Evangelium nach Thomas. Koptischer Text hg. u.übers. v. A. Guillaumont, H.-Ch. Puech, G. Quispel, W.Till u. Yassah Abd al Masih, Leiden 1959, 25, Log. 42. 